PROCEEDINGS OF THE

AMERICAN MATHEMATICAL SOCIETY

Volume 134, Number 5, Pages 1413-1423

S 0002-9939(05)08108-6

Article electronically published on October 7, 2005

\title{
CONVERGENCE OF CASCADE SEQUENCE ON THE HEISENBERG GROUP
}

\author{
HEPING LIU AND YU LIU
}

(Communicated by David R. Larson)

\begin{abstract}
The investigation of convergence of cascade sequence plays an important role in wavelet analysis on the Euclidean space and also in wavelet analysis on the Heisenberg group. This paper characterizes the $L^{p}\left(\mathbf{H}^{d}\right)(1 \leq$ $p \leq \infty)$-convergence of cascade sequence on the Heisenberg group in terms of the $p$-norm joint spectral radius of a collection of matrices associated with the refinement sequence and gives a sufficient condition.
\end{abstract}

\section{Introduction}

In the theory of wavelets, if one goes beyond the Euclidean space $\mathbf{R}^{d}$, the first and perhaps the most important case is the Heisenberg group $\mathbf{H}^{d}$. The theory of admissible (continuous) wavelets on the Heisenberg group was established in [6]. 10] gave a result on the existence of the orthogonal (discrete) wavelets on the Heisenberg group and showed that at most countably many solvable Lie groups are suitable for building multiresolution analysis. In [7] Peng constructed two kinds of orthogonal wavelets on the Heisenberg group. To our best knowledge, whether the Cohen's condition holds on the Heisenberg group has not been proved. Thus, the convergence of cascade sequence may be the only method to check the existence of refinable functions and to check the orthogonality of shifts of refinable functions on the Heisenberg group. Therefore, the investigation of the convergence of cascade sequence is important in wavelet analysis on the Heisenberg group. In this paper we shall investigate the convergence of cascade sequence in $L^{p}\left(\mathbf{H}^{d}\right)(1 \leq p \leq \infty)$ on the Heisenberg group. We partly extend the results of convergence of cascade sequence on the Euclidean space in 2 to the Heisenberg group.

The Heisenberg group $\mathbf{H}^{d}$ is a Lie group with the underlying manifold $\mathbf{R}^{d} \times \mathbf{R}^{d} \times$ $\mathbf{R}$ and the multiplication

$$
(x, y, t)\left(x^{\prime}, y^{\prime}, t^{\prime}\right)=\left(x+x^{\prime}, y+y^{\prime}, t+t^{\prime}+2 x^{\prime} y-2 x y^{\prime}\right),
$$

where $x=\left(x_{1}, \cdots, x_{d}\right), x^{\prime}=\left(x_{1}^{\prime}, \cdots, x_{d}^{\prime}\right), y=\left(y_{1}, \cdots, y_{d}\right), y^{\prime}=\left(y_{1}, \cdots, y_{d}\right) \in \mathbf{R}^{d}$, and $t^{\prime}, t \in \mathbf{R}$.

Received by the editors July 13, 2004 and, in revised form, December 13, 2004.

2000 Mathematics Subject Classification. Primary 40A30, 42C15, 39B99, 65F15.

Key words and phrases. Cascade sequence, refinement equations, joint spectral radius, wavelets, refinable functions.

This research was supported by the National Natural Science Foundation of China (No. 10371004) and the Specialized Research Fund for the Doctoral Program of Higher Education of China (No. 20030001107). 
Let $\Gamma=\left\{(m, n, l) \in \mathbf{H}^{d}: m, n, \in \mathbf{Z}^{d}, l \in \mathbf{Z}\right\}$ be a discrete subgroup of $\mathbf{H}^{d}$ satisfying $\alpha \Gamma \subset \Gamma$, where $\alpha$ is the homogeneous dilation of Heisenberg group defined by $\alpha(x, y, t)=(2 x, 2 y, 4 t)$. Let $\Gamma_{0}$ be a complete representative of distinct right cosets of $\Gamma / \alpha(\Gamma)$ such that $0 \in \Gamma_{0}$. It is easy to show that $[\alpha \Gamma, \Gamma]=2^{Q}$, where $Q=2(d+1)$ is the homogeneous dimension of Heisenberg group. For example, when $d=1$, we can choose

$$
\Gamma_{0}=\left\{\epsilon=\left(\epsilon_{1}, \epsilon_{2}, \epsilon_{3}\right): \epsilon_{1}, \epsilon_{2}=0, \text { or } 1 ; \epsilon_{3}=0,1,2 \text {, or } 3\right\} .
$$

The discrete subgroup $\Gamma$ acts on $\mathbf{H}^{d}$ by

$$
\begin{aligned}
U_{\gamma} f(x, y, t) & =U_{(m, n, l)} f(x, y, t)=f\left(\gamma^{-1}(x, y, t)\right) \\
& =f(x-m, y-n, t-l-2(n x-m y)),
\end{aligned}
$$

where $\gamma=(m, n, l) \in \Gamma$ and $f \in L^{p}\left(\mathbf{H}^{d}\right)$.

The dilation $\alpha$ acts on $L^{p}\left(\mathbf{H}^{d}\right)$ by

$$
\alpha f(x, y, t)=f(\alpha(x, y, t))=f(2 x, 2 y, 4 t), \quad f \in L^{p}\left(\mathbf{H}^{d}\right) .
$$

It is obvious that the operators $U_{\gamma}$ and $\alpha$ are not commutative, but we have $U_{\gamma} \alpha=$ $\alpha U_{\alpha(\gamma)}$.

The homogeneous norm on the Heisenberg group is defined as follows:

$$
|(x, y, t)|=\max \left(\left(|x|^{2}+|y|^{2}\right)^{\frac{1}{2}},|t|^{\frac{1}{2}}\right), \quad(x, y, t) \in \mathbf{H}^{d} .
$$

The theory of multiresolution analysis on the Heisenberg group has been established in [10. See [7] and [10] for the definition of multiresolution analysis on the Heisenberg group.

For a given multiresolution analysis on the Heisenberg group, there exists $2^{Q}-1$ wavelet functions $\left\{\psi^{\varepsilon}\right\}, \varepsilon \in \Gamma_{0}^{0}:=\Gamma_{0} \backslash\{0\}$, such that $\left\{D^{j} U_{\gamma} \psi^{\varepsilon}\right\}_{\varepsilon \in \Gamma_{0}^{0}, \gamma \in \Gamma, j \in \mathbf{Z}}$ forms an orthonormal (wavelet) basis for $L^{2}\left(\mathbf{H}^{d}\right)$, where $D^{j} f(\cdot)=2^{\frac{Q j}{2}} \alpha^{j} f(\cdot)$. This fact can be proved by the general approach in [1].

In order to investigate the convergence of cascade sequence, we shall consider the refinement equation

$$
\phi=\sum_{\gamma \in \Gamma} h(\gamma) \alpha U_{\gamma} \phi
$$

The nonzero solution of this equation is referred to as $(\alpha, h)$ refinable function and $h=(h(\gamma))_{\gamma \in \Gamma}$ is called a refinement sequence. In this paper, we assume that the refinable function $\phi$ is compactly supported and satisfies $\int_{\mathbf{H}^{d}} \phi(q) d q=1$. Also, we assume that the refinement sequence $h$ is finitely supported and satisfies the normalized condition

$$
\sum_{\gamma \in \Gamma} h(\gamma)=2^{Q}
$$

where $Q$ is the homogeneous dimension of $\mathbf{H}^{d}$.

Starting with a compactly supported function $\phi_{0}$, we define

$$
\phi_{n+1}=\sum_{\gamma \in \Gamma} h(\gamma) \alpha U_{\gamma} \phi_{n}, \quad n=0,1,2, \cdots
$$

The sequence (77) is called the cascade sequence associated with the refinement sequence $h$. We shall say that the cascade sequence converges (strongly) in $L^{p}\left(\mathbf{H}^{d}\right)$ if $\left\|\phi_{n}-\phi\right\|_{p} \rightarrow 0$ as $n \rightarrow \infty$. Many works are concerned with the convergence of cascade sequence on the Euclidean space. In [4] W. Lawton, S. L. Lee and 
Z. W. Shen investigated the $L^{2}\left(\mathbf{R}^{d}\right)$-convergence of cascade sequence in terms of the spectrum of the restricted transition operator. In 2 Bin Han and Rongqing Jia characterized the $L^{p}\left(\mathbf{R}^{d}\right)(1 \leq p \leq \infty)$-convergence of cascade sequence by the joint spectral radius. However, there are few papers concerned with this subject on the Heisenberg group. Lawton extended the theory of refinable functions to a connected nilpotent Lie group $\mathbf{G}$ and investigated the $L^{2}(\mathbf{G})$-convergence of cascade sequence in [5]. Based on these papers, we shall give a characterization of the strong convergence of cascade sequence on the Heisenberg group.

We denote by $\mathbf{N}, \mathbf{Z}, \mathbf{R}$ the set of the positive integer, the integer, and the real, respectively throughout this paper.

\section{Convergence of Cascade sequence on the Heisenberg group}

In order to solve the refinement equation (6), we start with a compactly supported function $\varphi \in L^{p}\left(\mathbf{H}^{d}\right)(1 \leq p \leq \infty)$ and use the iteration scheme $f_{n}=$ $T_{h}^{n} \varphi, n=0,1,2 \cdots$, where $T_{h}$ is the bounded operator on $L^{p}\left(\mathbf{H}^{d}\right)$ given by

$$
T_{h} \varphi=\sum_{\gamma \in \Gamma} h(\gamma) \alpha U_{\gamma} \varphi
$$

with the refinement sequence $h$ finitely supported on $\Gamma$.

Lemma 1. Let $h$ be a finitely supported refinement sequence on $\Gamma$, and let $T_{h}$ be the bounded operator defined by (8). Then

$$
T_{h}^{n} \varphi=\sum_{\gamma \in \Gamma} h_{n}(\gamma) \alpha^{n} U_{\gamma} \varphi
$$

where the sequences $h_{n}$ are given by

$$
h_{1}=h \quad \text { and } \quad h_{n}(\gamma)=\sum_{\beta \in \Gamma} h\left(\alpha(\beta)^{-1} \gamma\right) h_{n-1}(\beta) .
$$

Proof. This can be proved by induction on $n$. Indeed, (9) is valid for $n=1$. Suppose $n>1$ and (9) is true for $n-1$. Then by the induction hypothesis we have

$$
\begin{aligned}
T_{h}^{n} \varphi & =T_{h}^{n-1}\left(T_{h} \varphi\right) \\
& =\sum_{\gamma \in \Gamma} h_{n-1}(\gamma) \alpha^{n-1} U_{\gamma}\left(T_{h} \varphi\right) \\
& =\sum_{\gamma \in \Gamma} h_{n-1}(\gamma) \alpha^{n-1} U_{\gamma}\left(\sum_{\beta \in \Gamma} h(\beta) \alpha U_{\beta} \varphi\right) \\
& =\sum_{\beta \in \Gamma} \sum_{\gamma \in \Gamma} h_{n-1}(\gamma) h(\beta) \alpha^{n} U_{\alpha(\gamma) \beta} \varphi \\
& =\sum_{\beta \in \Gamma}\left(\sum_{\gamma \in \Gamma} h_{n-1}(\gamma) h\left(\alpha(\gamma)^{-1} \beta\right)\right) \alpha^{n} U_{\beta} \varphi \\
& =\sum_{\beta \in \Gamma} h_{n}(\beta) \alpha^{n} U_{\beta} \varphi .
\end{aligned}
$$

To describe our main result, we introduce the linear operator $A_{\varepsilon}\left(\varepsilon \in \Gamma_{0}\right)$ on $l_{0}(\Gamma)$ as follows:

$$
A_{\varepsilon} v(\gamma)=\sum_{\beta \in \Gamma} h\left(\alpha(\gamma) \varepsilon \beta^{-1}\right) v(\beta), \quad v \in l_{0}(\Gamma)
$$


where $l_{0}(\Gamma)$ is the space consisting of all finitely supported sequences on $\Gamma$.

Let $\Lambda$ be a finite collection of linear operators on a finite dimensional vector space $V$. For a linear operator $A$ on $V$, we define $\|A\|=\max _{\|v\|=1}\{\|A v\|\}$.

For a positive integer $n$ we denote by $\Lambda^{n}$ the Cartesian power of $\Lambda . \Lambda^{n}=$ $\left\{\left(A_{1}, \cdots, A_{n}\right): A_{1}, \cdots, A_{n} \in \Lambda\right\}$. When $n=0$, we interpret $\Lambda^{0}$ as the set $\{I\}$, where $I$ is the identity mapping on $V$. Let

$$
\left\|\Lambda^{n}\right\|_{\infty}=\max \left\{\left\|A_{1} \cdots A_{n}\right\|:\left(A_{1}, \cdots, A_{n}\right) \in \Lambda^{n}\right\} .
$$

Then the uniform spectral radius of $\Lambda$ is defined by

$$
\rho_{\infty}(\Lambda)=\lim _{n \rightarrow \infty}\left\|\Lambda^{n}\right\|_{\infty}^{\frac{1}{n}} .
$$

The uniform joint spectral radius was introduced by Rota and Strang in 8 .

The $p$-norm joint spectral radius of a finite collection of linear operators was introduced by Jia in 3 . We define, for $1 \leq p<\infty$,

$$
\left\|\Lambda^{n}\right\|_{p}=\left(\sum_{\left(A_{1}, \cdots, A_{n}\right) \in \Lambda^{n}}\left\|A_{1} \cdots A_{n}\right\|^{p}\right)^{\frac{1}{p}} .
$$

For $1 \leq p \leq \infty$, the $p$-norm joint spectral radius of $\Lambda$ is defined to be

$$
\rho_{p}(\Lambda)=\lim _{n \rightarrow \infty}\left\|\Lambda^{n}\right\|_{p}^{\frac{1}{n}}
$$

It can be easily verified that this limit exists, and

$$
\lim _{n \rightarrow \infty}\left\|\Lambda^{n}\right\|_{p}^{\frac{1}{n}}=\inf _{n \geq 1}\left\|\Lambda^{n}\right\|_{p}^{\frac{1}{n}} .
$$

Clearly, $\rho_{p}(\Lambda)$ is independent of the choice of the vector norm on $\nu$. If $\Lambda$ consists of a single linear operator $A$, then $\rho_{p}(\Lambda)=\rho(A)$, where $\rho(A)$ denotes the spectral radius of $A$, which is independent of $p$. If $\Lambda$ consists of more than one element, then $\rho_{p}(\Lambda)$ depends on $p$ in general. By some basic properties of $l^{p}$, we have that, for $1 \leq p \leq r \leq \infty$,

$$
(\# \Lambda)^{\frac{1}{r}-\frac{1}{p}} \rho_{p}(\Lambda) \leq \rho_{r}(\Lambda) \leq \rho_{p}(\Lambda),
$$

where $\# \Lambda$ denotes the number of elements in $\Lambda$. Furthermore, it is easily seen from the definition of the joint spectral radius that $\rho(A) \leq \rho_{\infty}(\Lambda)$ for any $A \in \Lambda$.

Lemma 2. Let $A_{\varepsilon}\left(\varepsilon \in \Gamma_{0}\right)$ be given by (11) and $v \in l_{0}(\Gamma)$. Suppose

$$
\xi=\alpha^{n}(\gamma) \alpha^{n-1}\left(\varepsilon_{n}\right) \cdots \alpha\left(\varepsilon_{2}\right) \varepsilon_{1},
$$

where $\varepsilon_{1}, \cdots, \varepsilon_{n} \in \Gamma_{0}, \gamma \in \Gamma$. Then

$$
\left(h_{n} * v\right)(\xi)=\left(A_{\varepsilon_{n}} \cdots A_{\varepsilon_{1}} v\right)(\gamma) .
$$

Proof. This can be proved by induction on $n$. For $n=1$, we have

$$
\left(h_{1} * v\right)(\xi)=\sum_{\beta \in \Gamma} h\left(\xi \beta^{-1}\right) v(\beta)=\sum_{\beta \in \Gamma} h\left(\alpha(\gamma) \varepsilon_{1} \beta^{-1}\right) v(\beta)=\left(A_{\varepsilon_{1}} v\right)(\gamma) .
$$

Hence (14) is true for $n=1$.

Suppose $n>1$ and (14) is valid for $n-1$. By (10) we have

$$
\left(h_{n} * v\right)(\xi)=\sum_{\beta \in \Gamma} h_{n}\left(\xi \beta^{-1}\right) v(\beta)=\sum_{\beta \in \Gamma} \sum_{\eta \in \Gamma} h_{n-1}(\eta) h\left(\alpha(\eta)^{-1} \xi \beta^{-1}\right) v(\beta) .
$$


Write $\xi=\alpha\left(\xi_{1}\right) \varepsilon_{1}$, where $\xi_{1}=\alpha^{n-1}(\gamma) \alpha^{n-2}\left(\varepsilon_{n}\right) \cdots \varepsilon_{2}$. Then

$$
\left(h_{n} * v\right)(\xi)=\sum_{\eta \in \Gamma} \sum_{\beta \in \Gamma} h_{n-1}(\eta) v(\beta) h\left(\alpha\left(\eta^{-1} \xi_{1}\right) \varepsilon_{1} \beta\right)=\sum_{\eta \in \Gamma} h_{n-1}(\eta) A_{\varepsilon_{1}} v\left(\eta^{-1} \xi_{1}\right) .
$$

By the induction hypothesis we obtain

$$
\left(h_{n} * v\right)(\xi)=\left(h_{n-1} * A_{\varepsilon_{1}} v\right)\left(\xi_{1}\right)=\left(A_{\varepsilon_{n}} \cdots A_{\varepsilon_{2}}\right)\left(A_{\varepsilon_{1}} v\right)(\gamma)=\left(A_{\varepsilon_{n}} \cdots A_{\varepsilon_{2}} A_{\varepsilon_{1}} v\right)(\gamma) .
$$

This completes the induction procedure.

A subspace $W$ of $V$ is said to be $\Lambda$-invariant if it is invariant under every operator $A$ in $\Lambda$. Let $X$ be a subset of $V$. The intersection of all $\Lambda$-invariant subspaces of $V$ containing $X$ is invariant, and we call it the minimal $\Lambda$-invariant subspace generated by $X$. This subspace is spanned by the set

$$
\left\{A_{1} \cdots A_{j} x: x \in X,\left(A_{1}, \cdots, A_{j}\right) \in \Lambda^{j}, j=0,1, \cdots\right\} .
$$

If $V$ is finite dimensional, then there exists a positive integer $k$ such that the set $\left\{A_{1} \cdots A_{j} x: x \in X,\left(A_{1}, \cdots, A_{j}\right) \in \Lambda^{j}, j=0,1, \cdots, k\right\}$ spans the minimal $\Lambda$ invariant subspace generated by $X$.

We define, for $1 \leq p<\infty$,

$$
\left\|\Lambda^{n} v\right\|_{p}=\left(\sum_{A_{1}, \cdots, A_{n} \in \Lambda^{n}}\left\|A_{1} \cdots A_{n} v\right\|^{p}\right)^{\frac{1}{p}}
$$

and, for $p=\infty$,

$$
\left\|\Lambda^{n} v\right\|_{\infty}=\max \left\{\left\|A_{1} \cdots A_{n} v\right\|:\left(A_{1}, \cdots, A_{n}\right) \in \Lambda^{n}\right\} .
$$

For $1 \leq p \leq \infty, l^{p}(\Gamma)$ denotes the Banach space of all sequences on $\Gamma$ such that $\|a\|_{p}<\infty$, where $\|a\|_{p}=\left(\sum_{\gamma \in \Gamma}|a(\gamma)|^{p}\right)^{\frac{1}{p}}(1 \leq p<\infty)$ and $\|a\|_{\infty}=\sup _{\gamma \in \Gamma}|a(\gamma)|$.

Let $v \in l_{0}(\Gamma)$. For $\gamma \in \Gamma$, we denote by $\nabla_{\gamma}$ the right difference operators on $l(\Gamma)$

$$
\nabla_{\gamma} v(\cdot)=v(\cdot)-v(\cdot \gamma)
$$

In the following lemma, the underlying vector norm on $l_{0}(\Gamma)$ is chosen to be the norm on $l^{p}(\Gamma)$.

Lemma 3. Let $\Lambda=\left\{A_{\varepsilon}, \varepsilon \in \Gamma_{0}\right\}$ and $v \in l_{0}(\Gamma)$. Then

$$
\left\|\Lambda^{n} v\right\|_{p}=\left\|h_{n} * v\right\|_{p}, \quad 1 \leq p \leq \infty,
$$

where $h_{n}$ is defined by (10) and $h_{n} * v$ is given by

$$
\left(h_{n} * v\right)(\gamma)=\sum_{\beta \in \Gamma} h_{n}(\beta) v\left(\beta^{-1} \gamma\right), \quad \gamma \in \Gamma .
$$

Proof. Suppose $\xi=\alpha^{n}(\gamma) \alpha^{n-1}\left(\varepsilon_{n}\right) \cdots \alpha\left(\varepsilon_{2}\right) \varepsilon_{1}$, where $\varepsilon_{1}, \cdots, \varepsilon_{n} \in \Gamma_{0}$ and $\gamma \in \Gamma$. Then by Lemma 2 we have

$$
\left(h_{n} * v\right)(\xi)=\left(A_{\varepsilon_{n}} \cdots A_{\varepsilon_{1}} v\right)(\gamma) .
$$

Hence (15) is true for $p=\infty$. When $1 \leq p<\infty$, we have

$$
\left\|h_{n} * v\right\|_{p}=\sum_{\xi \in \Gamma}\left|h_{n} * v(\xi)\right|^{p}=\sum_{\varepsilon_{1}, \cdots, \varepsilon_{n} \in \Gamma_{0}} \sum_{\gamma \in \Gamma}\left|A_{\varepsilon_{n}} \cdots A_{\varepsilon_{1}} v(\gamma)\right|^{p} .
$$

This verifies (15) for $1 \leq p<\infty$.

Suppose that $\Lambda=\left\{A_{\varepsilon}, \varepsilon \in \Gamma_{0}\right\}$. We claim that, for each $v \in l_{0}(\Gamma)$, the minimal $\Lambda$-invariant subspace generated by $v$ is finite dimensional. In order to establish this 
result, we shall introduce the concept of admissible sets. For a finite subset $K$ of $\Gamma$, recall that $l(K)$ is the linear subspace of $l_{0}(\Gamma)$ consisting of all sequences supported on $K$. A finite subset $K$ of $\Gamma$ is said to be admissible for $A$ if $l(K)$ is invariant under $A$ (see [2]). The following lemma shows that there exists a finite subset $K$ of $\Gamma$ such that $K$ contains the support of $v$ and is admissible for all $A_{\varepsilon}\left(\varepsilon \in \Gamma_{0}\right)$.

Lemma 4. Suppose $h$ is a sequence on $\Gamma$ with the support $\Omega=\{\gamma \in \Gamma: h(\gamma) \neq 0\}$ being finite. Let $A_{\varepsilon}$ be the linear operator on $l_{0}(\Gamma)$ given by (11). Then a finite subset $K$ of $\Gamma$ is admissible for $A=A_{0}$ if and only if

$$
\alpha^{-1}(\Omega K) \cap \Gamma \subseteq K .
$$

Consequently, for any finite subset $G$ of $\Gamma$, there exists a finite subset $K$ of $\Gamma$ such that $G \subseteq K$ and $K$ is admissible for all $A_{\varepsilon}, \varepsilon \in \Gamma_{0}$.

Proof. Suppose $K$ is admissible for $A$. Let $\gamma \in \alpha^{-1}(\Omega K) \cap \Gamma$. Then $\alpha(\gamma)=\eta \beta, \beta \in$ $K, \eta \in \Omega$. It follows that $A \delta_{\beta}(\gamma)=h\left(\alpha(\gamma) \beta^{-1}\right)=h(\eta) \neq 0$. Since $K$ is admissible for $A$, we have $A \delta_{\beta} \in l(K)$ and $\gamma \in K$. This shows that (16) is true.

Conversely, suppose (16) is true. Let $v \in l(K)$ and $\gamma \in \Gamma$. Then $A v(\gamma)=$ $\sum_{\beta \in \Gamma} h\left(\alpha(\gamma) \beta^{-1}\right) v(\beta) \neq 0$ implies that $\alpha(\gamma) \beta^{-1} \in \Omega$ for some $\beta \in K$. It follows that $\alpha(\gamma) \in \Omega K$. Therefore, $\gamma \in \alpha^{-1}(\Omega K) \cap \Gamma$, and $\gamma \in K$. This shows that $A$ maps $l(K)$ to $l(K)$. In other words, $K$ is admissible for $A$.

From the above proof we see that a subset $K$ of $\Gamma$ is admissible for $A_{\varepsilon}$ if and only if

$$
\alpha^{-1}\left(\Omega K \varepsilon^{-1}\right) \subseteq K .
$$

Now suppose $G$ is a finite subset of $\Gamma$. Let

$$
\begin{gathered}
H=\alpha G \cup \Omega \cup\{0\}, \\
K_{1}=\bigcup_{n=1}^{\infty}\left(\alpha^{-1}(H) \alpha^{-2}(H) \cdots \alpha^{-n}(H)\right), \\
K_{2}=\bigcup_{n=1}^{\infty}\left(\alpha^{-n}\left(\Gamma_{0}^{-1}\right) \cdots \alpha^{-2}\left(\Gamma_{0}^{-1}\right) \alpha^{-1}\left(\Gamma_{0}^{-1}\right)\right),
\end{gathered}
$$

and

$$
K=\left(K_{1} K_{2}\right) \cap \Gamma .
$$

In other words, $\gamma \in K$ if and only if $\gamma \in \Gamma$ and $\gamma=\gamma_{1} \gamma_{2}$, where $\gamma_{1} \in K_{1}, \gamma_{2} \in K_{2}$.

Since $0 \in H$ and $G \subseteq \alpha^{-1} H$, we have $G=G \cap \Gamma \subseteq \alpha^{-1} H \cap \Gamma \subseteq K$. Moreover, $\alpha^{-1}\left(\Omega K \varepsilon^{-1}\right) \cap \Gamma \subseteq\left(\alpha^{-1}(H) \alpha^{-1}\left(K_{1}\right) \alpha^{-1}\left(K_{2}\right) \alpha^{-1}\left(\varepsilon^{-1}\right)\right) \cap \Gamma \subseteq\left(K_{1} K_{2}\right) \cap \Gamma=K$.

Thus, $K$ satisfies (17). Hence $K$ is admissible for $A_{\varepsilon}, \varepsilon \in \Gamma_{0}$.

Lemma 5. Let $\Lambda$ be a finite collection of linear operators on a vector space $V$. Let $v \in V$, and let $V(v)$ be the minimal $\Lambda$-invariant subspace generated by $v$. If $V(v)$ is finite dimensional, then

$$
\lim _{n \rightarrow \infty}\left\|\Lambda^{n} v\right\|_{p}^{\frac{1}{n}}=\rho_{p}\left(\left.\Lambda\right|_{V(v)}\right) .
$$

We omit the proof of Lemma 5 because it is similar to that of Lemma 2.4 in [2]. 
Theorem 1. Let the refinement sequence $h$ be finitely supported. Let $\Lambda=\left\{A_{\varepsilon}: \varepsilon \in\right.$ $\left.\Gamma_{0}\right\}$, where $A_{\varepsilon}$ are the linear operators on $l_{0}(\Gamma)$ given by (11). Then for $v \in l_{0}(\Gamma)$,

$$
\lim _{n \rightarrow \infty}\left\|h_{n} * v\right\|_{p}^{\frac{1}{n}}=\rho_{p}\left(\left\{\left.A_{\varepsilon}\right|_{V(v)}: \varepsilon \in \Gamma_{0}\right\}\right),
$$

where $V(v)$ is the minimal $\Lambda$-invariant subspace generated by $v$. Moreover, if $W$ is the minimal $\Lambda$-invariant subspace generated by a finite set $Y$, then

$$
\rho_{p}\left(\left\{\left.A_{\varepsilon}\right|_{W}: \varepsilon \in \Gamma_{0}\right\}\right)=\max _{v \in Y}\left\{\lim _{n \rightarrow \infty}\left\|h_{n} * v\right\|_{p}^{\frac{1}{n}}\right\} .
$$

Proof. By Lemma [ $V(v)$ is finite dimensional, and so the relevant joint spectral radius in (20) is well defined.

By Lemma 3 we have

$$
\left\|h_{n} * v\right\|_{p}=\left\|\Lambda^{n} v\right\|_{p}, \quad 1 \leq p \leq \infty, n=1,2, \cdots .
$$

Applying Lemma 5 to the present situation, we obtain (19).

To prove the second part of the theorem, we note that $W$ is a finite sum of the linear subspace $V(v), v \in Y$. Hence

$$
\rho_{p}\left(\left\{\left.A_{\varepsilon}\right|_{W}: \varepsilon \in \Gamma_{0}\right\}\right)=\max _{v \in Y}\left\{\rho_{p}\left(\left\{\left.A_{\varepsilon}\right|_{V(v)}: \varepsilon \in \Gamma_{0}\right\}\right)\right\} .
$$

This, together with (19), verifies (20).

The next theorem gives a sufficient condition about the convergence of the cascade sequence on the Heisenberg group.

Theorem 2. Let $h$ be a finitely supported refinement sequence and $\sum_{\gamma \in \Gamma} h(\gamma)=$ $m=2^{Q}$. If

$$
\lim _{n \rightarrow \infty}\left\|\nabla_{j} h_{n}\right\|_{p}^{\frac{1}{n}}<m^{\frac{1}{p}}, j=1,2, \cdots, 2 d+1, \nabla_{j}=\nabla_{e_{j}},
$$

where $e_{1}, \cdots, e_{2 d+1}$ are the unit coordinate vectors in $\mathbf{R}^{2 d+1}$, then the cascade sequence associated with the refinement sequence $h$ converges in the $L^{p}$-norm $(1 \leq$ $p \leq \infty)$.

Proof. To simplify the statement we give the proof of the theorem in case of $d=1$. Of course, it holds when $d \neq 1$. Let $A_{\varepsilon}\left(\varepsilon \in \Gamma_{0}\right)$ be the linear operators on $l_{0}(\Gamma)$ given by (11) and $W$ the minimal invariant subspace of $A_{\varepsilon}\left(\varepsilon \in \Gamma_{0}\right)$ generated by $\nabla_{j} \delta(j=1,2,3)$. Then $W$ is finite dimensional, and by Theorem 1 we have

$$
\rho_{p}:=\rho_{p}\left(\left\{\left.A_{\varepsilon}\right|_{W}: \varepsilon \in \Gamma_{0}\right\}\right)=\max _{1 \leq j \leq 3}\left\{\lim _{n \rightarrow \infty}\left\|\nabla_{j} h_{n}\right\|_{p}^{\frac{1}{n}}\right\} .
$$

Thus (21) is equivalent to $\rho_{p}\left(\left\{\left.A_{\varepsilon}\right|_{W}: \varepsilon \in \Gamma_{0}\right\}\right)<m^{\frac{1}{p}}$.

We pick a compactly supported function $\phi$ in $L^{p}\left(\mathbf{H}^{1}\right)$ such that $\sum_{\gamma \in \Gamma} U_{\gamma} \phi=1$. Let $f_{n}=T_{h}^{n} \phi_{0}, g_{n}=T_{h}^{n} \phi$, where $T_{h}$ is the operator given by (8) and we can choose $\phi_{0}$ to be the characteristic function of the tile in $\mathbf{H}^{1}$ (see [9]) (in the case $p=\infty$, we assume that $\phi$ is continuous). Clearly, $\phi_{0}$ satisfies $\sum_{\gamma \in \Gamma} U_{\gamma} \phi_{0}=1$. Moreover, let $b_{n}$ be the sequence given by $b_{n}(\gamma)=\max _{1 \leq j \leq 3}\left|\nabla_{j} h_{n}(\gamma)\right|, \gamma \in \Gamma$. We claim that there exists a positive constant $C$ independent of $n$ such that

$$
\left\|f_{n+1}-g_{n}\right\|_{p} \leq C m^{-\frac{n}{p}}\left\|b_{n}\right\|_{p}, \quad 1 \leq p \leq \infty .
$$


If $\rho_{p}<m^{\frac{1}{p}}$, then we can find $r(0<r<1)$ such that $\rho_{p}<r m^{\frac{1}{p}}$. By the definition of $\rho_{p}$ and Theorem 1, we see that $\left\|b_{n}\right\|_{p}^{\frac{1}{n}}<r m^{\frac{1}{p}}$ for sufficiently large $n$. Hence, there exists a positive constant $C_{0}$ such that $\left\|b_{n}\right\|_{p} \leq C_{0}\left(r m^{\frac{1}{p}}\right)^{n}$ for all $n \geq 1$.

If we choose $\phi$ to be $\phi_{0}$, then it follows from (22) that $\left\|f_{n+1}-f_{n}\right\|_{p} \leq C C_{0} r^{n}$. This shows that the sequence $\left\{f_{n}\right\}$ converges to a function $f$ in the $L^{p}\left(\mathbf{H}^{1}\right)$-norm. Furthermore, (22) tells us that $\left\|f_{n+1}-g_{n}\right\|_{p} \rightarrow 0(n \rightarrow \infty)$.

On the other hand,

$$
\left\|g_{n+1}-f\right\|_{p} \leq\left\|f_{n+1}-g_{n}\right\|_{p}+\left\|f_{n+1}-f\right\|_{p} .
$$

Therefore, $\left\|g_{n+1}-f\right\|_{p} \rightarrow 0(n \rightarrow \infty)$. Thus, it suffices to prove (22).

For a sequence $\lambda \in l_{0}(\Gamma)$ and a subset $G$ of $\mathbf{H}^{1}$, we denote the supremum of $\lambda$ on the set $\Gamma \cap G$ by $\|\lambda\|_{\infty}(G)$. Let $\chi_{n}(\gamma)=\alpha^{-n}\left(\gamma[0,1)^{3}\right)$, for $n=1,2, \cdots, \gamma \in \Gamma$. We note that $\mathbf{H}^{1}=\bigcup_{\gamma \in \Gamma} \chi_{n}(\gamma)$. By (9) we have, for $q \in \mathbf{H}^{d}$,

$$
f_{n+1}(q)=\sum_{\beta} h_{n+1}(\beta) \alpha^{n+1} U_{\beta} \phi_{0}(q)
$$

and

$$
g_{n}(q)=\sum_{\eta} h_{n}(\eta) \alpha^{n} U_{\eta} \phi(q) .
$$

Since $\sum_{\beta} U_{\beta} \phi_{0}=1$ and $\sum_{\eta} U_{\eta} \phi=1$, it follows that

$$
f_{n+1}(q)-g_{n}(q)=\sum_{\eta} \sum_{\beta}\left[h_{n+1}(\beta)-h_{n}(\eta)\right] \alpha^{n} U_{\eta} \phi(q) \alpha^{n+1} U_{\beta} \phi_{0}(q) .
$$

In the above sum we only have to consider those terms for which $\alpha^{n} U_{\eta} \phi(q) \neq 0$ and $\alpha^{n+1} U_{\beta} \phi_{0}(q) \neq 0$.

Let $q \in \chi_{n}(\gamma)$, where $\gamma \in \Gamma$ is fixed for the time being. Suppose $\alpha^{n} U_{\eta} \phi(q) \neq 0$. Then we have $\gamma^{-1} \alpha^{n}(q) \in[0,1)^{3}$ and $\eta^{-1} \alpha^{n}(q) \in \operatorname{supp}(\phi)$. It follows that

$$
\eta=\gamma\left(\gamma^{-1} \alpha^{n}(q)\right)\left(\eta^{-1} \alpha^{n}(q)\right)^{-1} \in \gamma[0,1]^{3} \operatorname{supp}(\phi)^{-1} .
$$

Suppose $\alpha^{n+1} U_{\beta} \phi_{0}(q) \neq 0$. Then $\beta^{-1} \alpha^{n+1}(q) \in \operatorname{supp}\left(\phi_{0}\right)$. This, combining with $\eta^{-1} \alpha^{n}(q) \in \operatorname{supp}(\phi)$, yields

$$
\beta^{-1} \alpha(\eta)=\beta^{-1} \alpha^{n+1}(q)\left(\alpha\left(\eta^{-1} \alpha^{n}(q)\right)\right)^{-1} \in \operatorname{supp}\left(\phi_{0}\right) \alpha(\operatorname{supp}(\phi))^{-1} .
$$

We use the fact that $\sum_{\xi \in \Gamma} U_{\alpha(\xi)} h(\beta)=1$, for all $\beta \in \Gamma$. This will be proved later. Therefore, we have

$$
h_{n+1}(\beta)-h_{n}(\eta)=\sum_{\xi \in \Gamma} U_{\alpha(\xi)} h(\beta)\left[h_{n}(\xi)-h_{n}(\eta)\right] .
$$

We observe that $U_{\alpha(\xi)} h(\beta) \neq 0$ implies $\alpha(\xi)^{-1} \beta \in \operatorname{supp}(h)$. This, together with (25), gives

$$
\alpha\left(\xi^{-1} \eta\right)=\alpha\left(\xi^{-1}\right) \beta \beta^{-1} \alpha(\eta) \in \operatorname{supp}(h) \operatorname{supp}\left(\phi_{0}\right) \alpha(\operatorname{supp}(\phi))^{-1} .
$$

In light of (24) and (27), there exists a positive constant $M$ such that both $\eta$ and $\xi$ belong to $\gamma[-M, M]^{3}$, provided that $\alpha^{n} U_{\eta} \phi(q) \neq 0, \alpha^{n+1} U_{\beta} \phi_{0}(q) \neq 0$ and $U_{\alpha(\xi)} h(\beta) \neq 0$.

However, $h_{n}(\xi)-h_{n}(\eta)$ can be written as a sum of finite terms of the form $\nabla_{j} h_{n}(\theta)$, where $\theta \in \gamma[-A, A]^{3} \cap \Gamma, j=1,2,3$, and $A$ is a positive constant. Indeed, it follows from (27) that there exists a positive constant $B$ such that $\left|\xi^{-1} \eta\right| \leq B$. Let $\xi^{-1} \eta=\left(\varepsilon_{n 1}, \varepsilon_{n 2}, \varepsilon_{n 3}\right)$. We know $\left|h_{n}(\gamma)-h_{n}\left(\gamma e_{j}^{-1}\right)\right|$ can be written as the 
form $\left|h_{n}(\beta)-h_{n}\left(\beta e_{j}\right)\right|$, for $j=1,2,3$, and $\gamma, \beta \in \Gamma$. Without loss of generality, we assume that $\varepsilon_{n 1} \geq 0, \varepsilon_{n 2} \geq 0, \varepsilon_{n 3}+2 \varepsilon_{n 1} \varepsilon_{n 2} \geq 0$, where $\left(\varepsilon_{n 1}, \varepsilon_{n 2}, \varepsilon_{n 3}\right)=$ $\left(\varepsilon_{n 1}, 0,0\right)\left(0, \varepsilon_{n 2}, 0\right)\left(0,0, \varepsilon_{n 3}+2 \varepsilon_{n 1} \varepsilon_{n 2}\right)$. Since $\left|\xi^{-1} \eta\right| \leq B$, we have $\left|\varepsilon_{n 1}\right| \leq B$, $\left|\varepsilon_{n 2}\right| \leq B$, and $\left|\varepsilon_{n 3}\right| \leq B^{2}$. Hence, $\left|\varepsilon_{n 3}+2 \varepsilon_{n 1} \varepsilon_{n 2}\right| \leq 3 B^{2}$. Then $h_{n}(\xi)-h_{n}(\eta)$ can be expressed as the sum of $X_{1}, X_{2}$ and $X_{3}$, where

$$
\begin{aligned}
X_{1}=h_{n} & (\xi)-h_{n}(\xi(1,0,0))+h_{n}(\xi(1,0,0))-h_{n}(\xi(1,0,0)(1,0,0)) \\
& +\cdots \\
& +h_{n}\left(\xi\left(\varepsilon_{n 1}, 0,0\right)(-1,0,0)\right)-h_{n}\left(\xi\left(\varepsilon_{n 1}, 0,0\right)(-1,0,0)(1,0,0)\right), \\
X_{2}=h_{n} & \left(\xi\left(\varepsilon_{n 1}, 0,0\right)\right)-h_{n}\left(\xi\left(\varepsilon_{n 1}, 0,0\right)(0,1,0)\right) \\
& +h_{n}\left(\xi\left(\varepsilon_{n 1}, 0,0\right)(0,1,0)\right)-h_{n}\left(\xi\left(\varepsilon_{n 1}, 0,0\right)(0,1,0)(0,1,0)\right) \\
& +\cdots \\
& +h_{n}\left(\xi\left(\varepsilon_{n 1}, \varepsilon_{n 2}-1,2 \varepsilon_{n 1}-2 \varepsilon_{n 1} \varepsilon_{n 2}\right)\right) \\
& -h_{n}\left(\xi\left(\varepsilon_{n 1}, \varepsilon_{n 2}-1,2 \varepsilon_{n 1}-2 \varepsilon_{n 1} \varepsilon_{n 2}\right)(0,1,0)\right), \\
X_{3}=h_{n} & \left(\left(\varepsilon_{n 1}, \varepsilon_{n 2},-2 \varepsilon_{n 1} \varepsilon_{n 2}\right)\right)-h_{n}\left(\left(\varepsilon_{n 1}, \varepsilon_{n 2},-2 \varepsilon_{n 1} \varepsilon_{n 2}\right)(0,0,1)\right) \\
& +h_{n}\left(\left(\varepsilon_{n 1}, \varepsilon_{n 2}, 1-2 \varepsilon_{n 1} \varepsilon_{n 2}\right)\right)-h_{n}\left(\left(\varepsilon_{n 1}, \varepsilon_{n 2}, 1-2 \varepsilon_{n 1} \varepsilon_{n 2}\right)(0,0,1)\right) \\
& +\cdots \\
& +h_{n}\left(\left(\varepsilon_{n 1}, \varepsilon_{n 2}, \varepsilon_{n 3}-1\right)\right)-h_{n}\left(\left(\varepsilon_{n 1}, \varepsilon_{n 2}, \varepsilon_{n 3}-1\right)(0,0,1)\right) .
\end{aligned}
$$

It follows from the above discussion that there exists a positive constant $A$ such that the elements with the form $\xi\left(\gamma_{1}, 0,0\right) e_{1}, \xi\left(\gamma_{1}, \gamma_{2}, 0\right) e_{2}$ and $\xi\left(\gamma_{1}, \gamma_{2}, \gamma_{3}\right) e_{3}$ satisfy that their norms are less than $A$. Therefore, $h_{n}(\xi)-h_{n}(\eta)$ can be written as a sum of finite terms of the form $\nabla_{j} h_{n}(\theta)$, where $\theta \in \gamma[-A, A]^{3} \cap \Gamma$ and $j=1,2,3$.

Therefore, (26) tells us that there exists a positive constant $C_{1}$ independent of $n$ such that

$$
\left|h_{n+1}(\beta)-h_{n}(\eta)\right| \leq C_{1}\left\|b_{n}\right\|_{\infty}\left(\gamma[-A, A]^{3} \cap \Gamma\right),
$$

provided $\alpha^{n} U_{\eta} \phi(q) \alpha^{n+1} U_{\beta} \phi_{0}(q) \neq 0$ for $q \in \chi_{n}(\gamma)$. Since $\sum_{\beta \in \Gamma}\left|\alpha^{n} U_{\beta} \phi_{0}(q)\right|=1$, by (23) and (28) we obtain

$$
\left|f_{n+1}(q)-g_{n}(q)\right| \leq C_{1} \alpha^{n}|\phi|^{0}(q)\left\|b_{n}\right\|_{\infty}\left(\gamma[-A, A]^{3} \cap \Gamma\right),
$$

where $|\phi|^{0}(q)=\sum_{\gamma \in \Gamma}\left|U_{\gamma} \phi(q)\right|, q \in \mathbf{H}^{1}$.

In the case $p=\infty, \phi$ is a continuous function with compact support; hence, there exists a positive constant $C_{2}$ such that $|\phi|^{0}(q) \leq C_{2}$ for all $q \in \mathbf{H}^{1}$. It follows from (29) that $\left\|f_{n+1}-g_{n}\right\|_{\infty} \leq C_{1} C_{2}\left\|b_{n}\right\|_{\infty}$. This proves (22) for the case $p=\infty$.

For $1 \leq p<\infty$, we deduce from (29) that

$$
\int_{\chi_{n}(\gamma)}\left|f_{n+1}(q)-g_{n}(q)\right|^{p} d q \leq C_{1}^{p} \int_{\chi_{n}(\gamma)}\left[\alpha^{n}|\phi|^{0}(q)\right]^{p} d q \sum_{\eta \in \gamma[-A, A]^{3} \cap \Gamma}\left|b_{n}(\eta)\right|^{p} .
$$

Since $\phi$ is compactly supported, we have

$$
|\phi|^{0}(q)=\sum_{\eta \in \Gamma \bigcap[0,1)^{3}(\operatorname{supp}(\phi))^{-1}}\left|U_{\eta} \phi(q)\right|, \quad q \in[0,1)^{3} .
$$

Hence, $C_{3}:=\int_{[0,1)^{3}}|\phi|^{0}(q)^{p} d q<\infty$. Consequently,

$$
\int_{\chi_{n}(\gamma)}\left[\alpha^{n}|\phi|^{0}(q)\right]^{p} d q=m^{-n} \int_{\gamma[0,1)^{3}}\left[|\phi|^{0}(q)\right]^{p} d q=C_{3} m^{-n} .
$$


Finally, we obtain

$$
\begin{aligned}
\left\|f_{n+1}-g_{n}\right\|_{p}^{p} & \leq \sum_{\gamma \in \Gamma} \int_{\chi_{n}(\gamma)}\left|f_{n+1}(q)-g_{n}(q)\right|^{p} d q \\
& \leq C_{1}^{p} C_{3} m^{-n} \sum_{\gamma \in \Gamma} \sum_{\eta \in \gamma[-A, A]^{3} \cap \Gamma}\left|b_{n}(\eta)\right|^{p} .
\end{aligned}
$$

However,

$$
\sum_{\gamma \in \Gamma} \sum_{\eta \in \gamma[-A, A]^{3} \cap \Gamma}\left|b_{n}(\eta)\right|^{p}=\sum_{\eta \in \Gamma}\left|b_{n}(\eta)\right|^{p} \sum_{\gamma \in \eta[-A, A]^{3} \cap \Gamma} 1=C_{4} \sum_{\eta \in \Gamma}\left|b_{n}(\eta)\right|^{p},
$$

where $C_{4}$ is the number of the element of $\eta[-A, A]^{3} \cap \Gamma$.

Therefore, there exists a positive constant $C_{5}$ such that

$$
\left\|f_{n+1}-g_{n}\right\|_{p} \leq C_{5} m^{\frac{-n}{p}}\left\|b_{n}\right\|_{p}
$$

This proves (22).

At last, we prove that $\sum_{\xi \in \Gamma} U_{\alpha(\xi)} h(\beta)=1$, for all $\beta \in \Gamma$. It is sufficient to prove $\sum_{\xi \in \Gamma} U_{\alpha(\xi)} h(\beta)=1$, for all $\beta \in \Gamma_{0}$.

Note that $\Gamma$ is the disjoint union of $\alpha(\Gamma) \varepsilon\left(\varepsilon \in \Gamma_{0}\right)$. Since $\sum_{\gamma \in \Gamma} h(\gamma)=16$, $\sum_{\varepsilon \in \Gamma_{0}} \sum_{\beta \in \Gamma} U_{\alpha(\beta)} h(\varepsilon)=16$. Therefore, we only need to prove that

$$
\sum_{\beta \in \Gamma} U_{\alpha(\beta)} h(\varepsilon)=\sum_{\beta \in \Gamma} U_{\alpha(\beta)} h(0), \quad \forall \varepsilon \in \Gamma_{0} .
$$

We know from (10) that

$$
\begin{aligned}
\sum_{\beta \in \Gamma} h_{n}(\beta) & =\sum_{\beta \in \Gamma} \sum_{\gamma \in \Gamma} h\left(\alpha(\gamma)^{-1} \beta\right) h_{n-1}(\gamma) \\
& =\sum_{\beta \in \Gamma} h\left(\alpha(\gamma)^{-1} \beta\right)\left(\sum_{\gamma \in \Gamma} h_{n-1}(\gamma)\right)=16 \sum_{\gamma \in \Gamma} h_{n-1}(\gamma) .
\end{aligned}
$$

We deduce by the induction on $n$ that $\sum_{\beta \in \Gamma} h_{n}(\beta)=16^{n}$. Moreover,

$$
\begin{aligned}
\sum_{\beta \in \Gamma} U_{\alpha(\beta)} h_{n}(\gamma) & =\sum_{\beta \in \Gamma} \sum_{\eta \in \Gamma} U_{\alpha(\beta)} U_{\alpha(\eta)} h(\gamma) h_{n-1}(\eta) \\
& =\sum_{\beta \in \Gamma} U_{\alpha(\beta)} h(\gamma)\left(\sum_{\eta \in \Gamma} h_{n-1}\left(\beta^{-1} \eta\right)\right) \\
& =16^{n-1} \sum_{\beta \in \Gamma} U_{\alpha(\beta)} h(\gamma) .
\end{aligned}
$$

Therefore,

$$
\sum_{\beta \in \Gamma} U_{\alpha(\beta)}[h(\gamma)-h(0)]=16^{-(n-1)} \sum_{\beta \in \Gamma}\left[U_{\alpha(\beta)} h_{n}(\gamma)-U_{\alpha(\beta)} h_{n}(0)\right] .
$$

It follows that

$$
\left|\sum_{\beta \in \Gamma}\left(U_{\alpha(\beta)} h_{n}(\gamma)-U_{\alpha(\beta)} h_{n}(0)\right)\right| \leq 16^{-(n-1)}\left\|\nabla_{\gamma} h_{n}\right\|_{1} .
$$

Since $\gamma \in \Gamma_{0},\left\|\nabla_{\gamma} h_{n}\right\|_{1}$ is less than a finite sum of $\left\|\nabla_{j} h_{n}\right\|_{1}(j=1,2,3)$ (it is in the same way we write $h_{n}(\xi)-h_{n}(\eta)$ as a finite sum of $\left.\nabla_{j} h_{n}(\theta)\right)$. 
If (21) holds for $p=1$, then we have that $16^{-(n-1)}\left\|\nabla_{\gamma} h_{n}\right\|_{1} \rightarrow 0$ as $n \rightarrow \infty$. If (21) holds for $1<p \leq \infty$, then it follows from (13) that

$$
\rho_{1}\left(\left\{\left.A_{\varepsilon}\right|_{W}: \varepsilon \in \Gamma_{0}\right\}\right) \leq 16^{1-\frac{1}{p}} \rho_{p}\left(\left\{\left.A_{\varepsilon}\right|_{W}: \varepsilon \in \Gamma_{0}\right\}\right)<16^{1-\frac{1}{p}} 16^{\frac{1}{p}}=16,
$$

because $\#\left\{\left.A_{\varepsilon}\right|_{W}: \varepsilon \in \Gamma_{0}\right\}=16$. Thus, (21) is still valid for $p=1$, and we have that $16^{-(n-1)}\left\|\nabla_{\gamma} h_{n}\right\|_{1} \rightarrow 0$ as $n \rightarrow \infty$.

Therefore, this proves $\sum_{\xi \in \Gamma} U_{\alpha(\xi)} h(\beta)=1$, for all $\beta \in \Gamma_{0}$.

Remark. In Theorem 2 we only give a sufficient condition to characterize the convergence of cascade sequence on the Heisenberg group, which is different from that of the Euclidean space (see [2]).

\section{ACKNOWLEDGEMENTS}

The authors would like to thank Professor Lizhong Peng, Rongqing Jia and R. Strichartz for providing us with their literatures on this subject.

\section{REFERENCES}

[1] L. Baggett, A. Carey, W. Moran, and P. Ohring, General existence theorems for orthonormal wavelets, an abstract approach, publications of the Research Institute of Mathematical science, Kyoto University, 31 (1995) no. 1, 95-111. MR.1317525 (96c:42060)

[2] B. Han and R.Q. Jia, Multivariate refinement equations and convergence of subdivision schemes, SIAM. J. Math. Anal. 29 (1998), no.5, 1177-1199. MR1618691 (99f:41018)

[3] R. Q. Jia, Subdivision Scheme in $L^{p}$ spaces, Adv. Comput. Math. 3 (1995), 309-341. MR:1339166 (96d:65028)

[4] W. Lawton, S. L. Lee, and Z. W. Shen, Convergence of multidimensional cascade algorithm, Numer. Math. 78 (1997), no. 3, 427-438. MR.1603354 (98k:41027)

[5] W. Lawton, Infinite convolution products and refinable distributions on Lie groups, Trans. Amer. Math. Soc. 352 (2000), 2913-2936. MR1638258(2000j:43002)

[6] H. P. Liu and L. Z. Peng, Admissible wavelets associated with the Heisenberg Group, Pacific J. Math. 180 (1997), 101-123. MR1474896 (99d:42065)

[7] L. Z. Peng, Wavelets on the Heisenberg Group, Geometry and Nonlinear Partial Differential Equations, AMS/IP Studies in Advanced Mathematics, 29 (2002), 123-131. MR 1926440 (2003j:43016)

[8] G. C. Rota and W. G. Strang, A note on the joint spectral radius, Indag. Math. 22 (1960), 379-381. MR147922(26:5434)

[9] R. S. Strichartz, Self-similarity on nilpotent Lie group, Contemp. Math. 140 (1992), 123-157. MR.1197594 (94e:43011)

[10] Q. D. Yang, Multiresolution analysis on non-abelian locally compact groups, Ph.D. Thesis, Dept. of Math., Univ. of Saskatchewan, 1999.

Lmam, School of Mathematical Sciences, Peking University, Beijing 100871, PeoPLE'S REPUBLIC OF CHINA

E-mail address: hpliu@math.pku.edu.cn

Lmam, School of Mathematical Sciences, Peking University, Beijing 100871, PeoPLE'S Republic OF ChinA

E-mail address: liuyu@math.pku.edu.cn 